\title{
Interstellar Plunging Waves: ALMA Resolves the Physical Structure of Nonstationary MHD Shocks
}

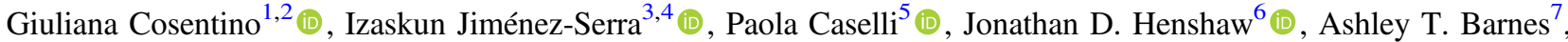 \\ Jonathan C. $\operatorname{Tan}^{8,9}$ (10), Serena Viti ${ }^{1}$ (i), Francesco Fontani ${ }^{10}$ (i), and Benjamin $\mathrm{Wu}^{11}$ (1) \\ ${ }^{1}$ Department of Physics and Astronomy, University College London, Gower Street, London WC1E6BT, UK; giuliana.cosentino.15@ucl.ac.uk \\ ${ }^{2}$ European Southern Observatory, Karl-Schwarzschild-Strasse 2, D-85748 Garching, Germany \\ ${ }^{3}$ Departamento de Astrofísica, Centro de Astrobiologia, E-28850 Torrejón de Ardoz, Madrid, Spain \\ ${ }^{4}$ School of Physics \& Astronomy, Queen Mary University of London, Mile End Road, London E1 4NS, UK \\ ${ }_{5}$ Max-Planck Institute for Extraterrestrial Physics, Gießenbachstrasse 2, D-85748 Garching, Germany \\ ${ }^{6}$ Max-Planck Instiutute for Astronomy, Kónigstuhl 17, D-69117 Heidelberg, Germany \\ ${ }^{7}$ Argelander-Institut für Astronomie Auf dem Hügel 71, D-53121 Bonn, Germany \\ ${ }^{8}$ Space, Earth and Environment Department, Chalmers University of Technology, Chalmersplatsen 4, SE-41296 Göteborg, Sweden \\ ${ }^{9}$ Department of Astronomy, University of Virginia, Charlottesville, VA, USA \\ ${ }^{10}$ INAF-Osservatorio Astrofisico di Arcetri, Largo E. Fermi 2, I-50125 Firenze, Italy \\ ${ }^{11}$ National Astronomical Observatory of Japan, Yubinbango 181-8588 Tokio, Mitaka, Osawa 2-21-1, Japan \\ Received 2019 June 24; revised 2019 August 1; accepted 2019 August 6; published 2019 August 21
}

\begin{abstract}
Magnetohydrodynamic (MHD) shocks are violent events that inject large amounts of energy in the interstellar medium dramatically modifying its physical properties and chemical composition. Indirect evidence for the presence of such shocks has been reported from the especial chemistry detected toward a variety of astrophysical shocked environments. However, the internal physical structure of these shocks remains unresolved since their expected spatial scales are too small to be measured with current instrumentation. Here we report the first detection of a fully spatially resolved, MHD shock toward the infrared dark cloud (IRDC) G034.77-00.55. The shock, probed by silicon monoxide ( $\mathrm{SiO}$ ) and observed with the Atacama Large Millimeter/submillimeter Array (ALMA), is associated with the collision between the dense molecular gas of the cloud and a molecular gas flow pushed toward the IRDC by the nearby supernova remnant (SNR) W44. The interaction is occurring on subparsec spatial scales thanks to the enhanced magnetic field of the SNR, making the dissipation region of the MHD shock large enough to be resolved with ALMA. Our observations suggest that molecular flow-flow collisions can be triggered by stellar feedback, inducing shocked molecular gas densities compatible with those required for massive star formation.
\end{abstract}

Key words: ISM: clouds (G034.77-00.55) - ISM: molecules - ISM: supernova remnants (W44) - shock waves

\section{Introduction}

Interstellar magnetohydrodynamic (MHD) shocks are known to exist in a wide variety of astrophysical environments such as jets and molecular outflows (Martín-Pintado et al. 1992; Caselli et al. 1997; Girart \& Acord 2001), stellar winds (Wilkin 1996; Babel \& Montmerle 1997), and supernovae explosions (Gotthelf et al. 2001; Grefenstette et al. 2014; Miceli et al. 2019). Depending on the propagation speed, pre-shock gas density, and magnetic field strength, MHD shocks can be either $\mathrm{J}$ type (from jump, fast shocks with $v_{\mathrm{s}} \geqslant 45-50 \mathrm{~km} \mathrm{~s}^{-1}$ and with a discontinuity in the gas physical properties) or $\mathrm{C}$ type (more gentle interactions found in molecular clouds with lowionization fractions, where the gas physical properties change in a continuous manner; Draine 1980; Draine et al. 1983; Neuufeld \& Dalgarno 1989). If the MHD shock has not attained the steady state, theory predicts the presence of a J-type discontinuity within the structure of the shock at the early stages of its propagation (the piston), which later on evolves into a C-type shock (a nonstationary CJ-type shock; Chiéze et al. 1998; Flower \& Pineau de Forêts 2003; Lesaffre et al. 2004).

In star-forming regions, MHD shocks are expected to develop across spatial scales of $\sim 10^{14}-10^{15} \mathrm{~cm}\left(\sim 0.05^{\prime \prime}-0.5^{\prime \prime}\right.$ at a distance of $140 \mathrm{pc}$; Gusdorf et al. 2008), too small to be easily resolved with current instrumentation. However, interstellar shocks are also predicted to arise when large-scale flows of molecular gas are pushed to collide. In such collisions, the shock occurs across parsec/subparsec spatial scales $\left(\sim 3 \times 10^{17}-3 \times 10^{18} \mathrm{~cm}\right)$, sufficient to spatially resolve its physical and dynamical evolution (Inutsuka et al. 2015; Wu et al. 2015).

In this Letter, we report the first images of the internal physical structure of a nonstationary CJ-type shock. The emission of the typical shock tracer $\mathrm{SiO}$ has been mapped with Atacama Large Millimeter/submillimeter Array (ALMA) toward the IRDC G034.77-00.55 (hereafter G034), which is located at $2.9 \mathrm{kpc}$ in a highly dynamical environment between the H II region G034.8-00.7 and the supernova remnant (SNR) W44. The interaction between G034 and W44, located at the same distance, has long been established (e.g., Claussen et al. 1997; Seta et al. 1998, 2004; Ortega et al. 2007; Yoshiike et al. 2013; Ranasinghe \& Leahy 2018), making G034 an ideal target for MHD shock investigation. Our ALMA images show that a molecular gas flow pushed by the expanding shell of W44 is interacting with a dense molecular ridge in $\mathrm{G} 034$ forcing the molecular flow to decelerate and plunge onto the infrared dark cloud (IRDC). This interaction enhances the density of the gas in the ridge to levels required for the formation of the most massive stars. 


\section{Observations}

We used ALMA Band 3 during Cycle 4 (PI: I. JimenezSerra) to map the $\mathrm{SiO} J=2 \rightarrow 1$ transition $(86.85 \mathrm{GHz})$ toward G034. Observations were performed using the ALMA Compact Array (11 antennas) plus 45 antennas of the $12 \mathrm{~m}$ array (baseline 15-331 m; uv distance 4.3-9.6 k $\lambda$ ). Observations were performed in dual polarization mode, using a spectral bandwidth of $58.594 \mathrm{MHz}$. The sources J1751+0939 and $\mathrm{J} 1851+0035$ were used as calibrators. We reproduced the two calibrated data sets by running the original pipeline reduction scripts and used the task uvcontsub in CASA version 4.7.2 $2^{12}$ to subtract the continuum emission previously estimated from line-free channels. The two continuum-subtracted data cubes were combined using the task concat. We generated the $\mathrm{SiO}$ line-only final image using the task clean with Briggs weighting (robust parameter 0.5), phase center $\alpha(\mathrm{J} 2000)=18^{\mathrm{h}} 56^{\mathrm{m}} 41^{\mathrm{s}} \mathrm{7}, \delta(\mathrm{J} 2000)=1^{\circ} 23^{\prime} 25^{\prime \prime}$, and velocity resolution $0.1 \mathrm{~km} \mathrm{~s}^{-1}(\sim 30 \mathrm{kHz})$. The final high angular resolution images have a synthesized beam of 3 ." $5 \times 2$." 5 , position angle $(\mathrm{PA})=84^{\circ} .27$, and noise level of $10 \mathrm{mJy}$ beam $^{-1}$. By comparing the ALMA and IRAM $30 \mathrm{~m}$ spectra extracted toward the position of the $\mathrm{SiO}$ peak detected by Cosentino et al. (2018) in this cloud, we find that no significant flux is missing in the $12 \mathrm{~m}+\mathrm{ACA} \mathrm{SiO}$ image. The $3 \mathrm{~mm}$ continuum map obtained with the $12 \mathrm{~m}$ array does not show any continuum pointlike source spatially associated with $\mathrm{SiO}$ and above the $3 \sigma$ level $\left(1 \sigma=66 \mu \mathrm{Jy}_{\text {beam }}{ }^{-1}\right)$.

Observations of the $\mathrm{C}^{18} \mathrm{O} J=1 \rightarrow 0(109.78 \mathrm{GHz})$ and $J=2 \rightarrow 1(219.56 \mathrm{GHz})$ lines were carried out with the IRAM $30 \mathrm{~m}$ telescope in 2012 July. The On-The-Fly maps were obtained using the off position $\left(-240^{\prime \prime},-40^{\prime \prime}\right)$ and a dump speed of $6^{\prime \prime} \mathrm{s}^{-1}$. Pointing accuracy was $\leqslant 3^{\prime \prime}$ and line calibrations were performed on the source G34.4+0.3. The fast Fourier Transform Spectrometer (FTS; $4 \mathrm{GHz}$ full bandwidth) provided a spectral resolution of $200 \mathrm{kHz}$, i.e., $0.54 \mathrm{~km} \mathrm{~s}^{-1}$ at $109.78 \mathrm{GHz}$ and $0.27 \mathrm{~km} \mathrm{~s}^{-1}$ at $219.56 \mathrm{GHz}$. The final data cubes, created using the CLASS software within GILDAS, ${ }^{13}$ have a spatial resolution and beam efficiency of $24^{\prime \prime}$ and 0.78 for $\mathrm{C}^{18} \mathrm{O}(1-0)$ and $12^{\prime \prime}$ and 0.63 for $\mathrm{C}^{18} \mathrm{O}(2-1)$. The final $\mathrm{rms}$ is $0.1 \mathrm{~K}$ for both spectra.

\section{Results}

\subsection{SiO ALMA Data: Moment Maps and Position-Velocity (PV) Diagrams}

In Figure 1, we present the three-color image of G034 (black circle) located between W44 (blue circle) and the H II region G034.758-00.681 (green circle). Red is $24 \mu \mathrm{m}$ emission (Spitzer MIPSGAL; Carey et al. 2009), green is $8 \mu \mathrm{m}$ emission (Spitzer GLIMPSE; Churchwell et al. 2009), and blue shows a combined Jansky Very Large Array (JVLA) and Green Bank Telescope (GBT) $21 \mathrm{~cm}$ continuum map (THOR survey; Beuther et al. 2016). The white square indicates the extent of the ALMA mosaic. The molecular gas around W44 is expanding at a velocity of $\sim 11-13 \mathrm{~km} \mathrm{~s}^{-1}$ (Sashida et al. 2013), although in regions close to G034 it shows terminal velocities $\sim 20-25 \mathrm{~km} \mathrm{~s}^{-1}$ (see region W44F; Anderl et al. 2015a). The molecular gas outside W44 moves with

\footnotetext{
12 https://casa.nrao.edu/

13 https://www.iram.fr/IRAMFR/GILDAS/
}

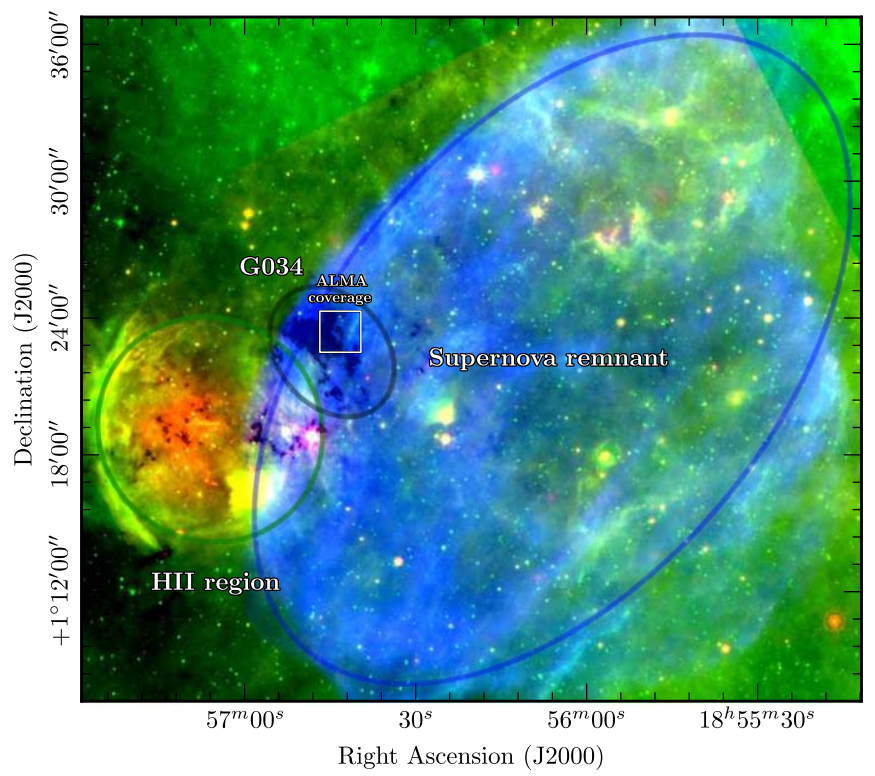

Figure 1. Three-color image of G034 showing its position (black circle) between W44 (blue circle) and G034.758-00.681 (green circle). Red is $24 \mu \mathrm{m}$ emission (Spitzer MIPSGAL; Carey et al. 2009), green is $8 \mu \mathrm{m}$ emission (Spitzer GLIMPSE; Churchwell et al. 2009), and blue shows a combined JVLA +GBT $21 \mathrm{~cm}$ continuum map (THOR survey; Beuther et al. 2016). The white square indicates the ALMA mosaic area and the gray shadow corresponds to $A_{v} \geqslant 20 \mathrm{mag}$.

$v_{\mathrm{LSR}} \sim 45-50 \mathrm{~km} \mathrm{~s}^{-1}$ (i.e., distances $S \geqslant 1$ in Figures 6 and 7 of Sashida et al. 2013).

Figure 2 reports the integrated intensity map (black contours; velocity range $36.6-47.6 \mathrm{~km} \mathrm{~s}^{-1}$ ) and the velocity moment 1 map (red scale) of the $\mathrm{SiO}$ emission measured toward G034 with ALMA. These maps appear superimposed on the $A_{\mathrm{v}}=20 \mathrm{mag}$ visual extinction level of the cloud (Kainulainen $\&$ Tan 2013), which shows a dense ridge toward the northwest of G034. The SiO emission is organized into two plane-parallel structures: a bright elongation peaking outside the ridge at redshifted velocities with respect to the radial velocity of the IRDC $\left(v_{\mathrm{LSR}}=43 \mathrm{~km} \mathrm{~s}^{-1}\right)$ and a fainter elongation found into the ridge between $\sim 36-42 \mathrm{~km} \mathrm{~s}^{-1}$. The $\mathrm{SiO}$ channel maps (Figure 3) reveal that shifting from 46 to $39 \mathrm{~km} \mathrm{~s}^{-1}$, SiO first appears in a region outside the $A_{\mathrm{v}} \sim 20 \mathrm{mag}$ ridge, to progressively move toward the cloud bending and spreading onto it (as if it had impacted).

To analyze the velocity structure of the $\mathrm{SiO}$ shocked gas, we have extracted PV diagrams along two representative directions perpendicular to the observed $\mathrm{SiO}$ elongations, $\mathrm{PV}$ north and PV south (blue arrows in Figure 2). Figure 4 shows that the deceleration of the gas is occurring in two steps: a first almost vertical velocity decrease of $\sim 2-3 \mathrm{~km} \mathrm{~s}^{-1}$ within a spatial width lower than the angular resolution $(\leqslant 2$ !" 5$)$, and a second and shallower deceleration of $\sim 4-5 \mathrm{~km} \mathrm{~s}^{-1}$ measured across $\sim 10^{\prime \prime}-20^{\prime \prime}$. This resembles the predicted velocity structure of CJ-type shocks (Section 4 and Chiéze et al. 1998; Lesaffre et al. 2004). The comparison between the SiO PV diagrams and the visual extinction profiles of G034 along the PV directions reveals that the observed $\mathrm{SiO}$ spatially coincides with extinction peaks across the ridge (bottom brown curves in Figure 4). This is consistent with the idea that the two shocked plane-parallel structures (probed by $\mathrm{SiO}$ ) appear once the molecular gas expanding from W44 encounters the highdensity wall of the ridge. 


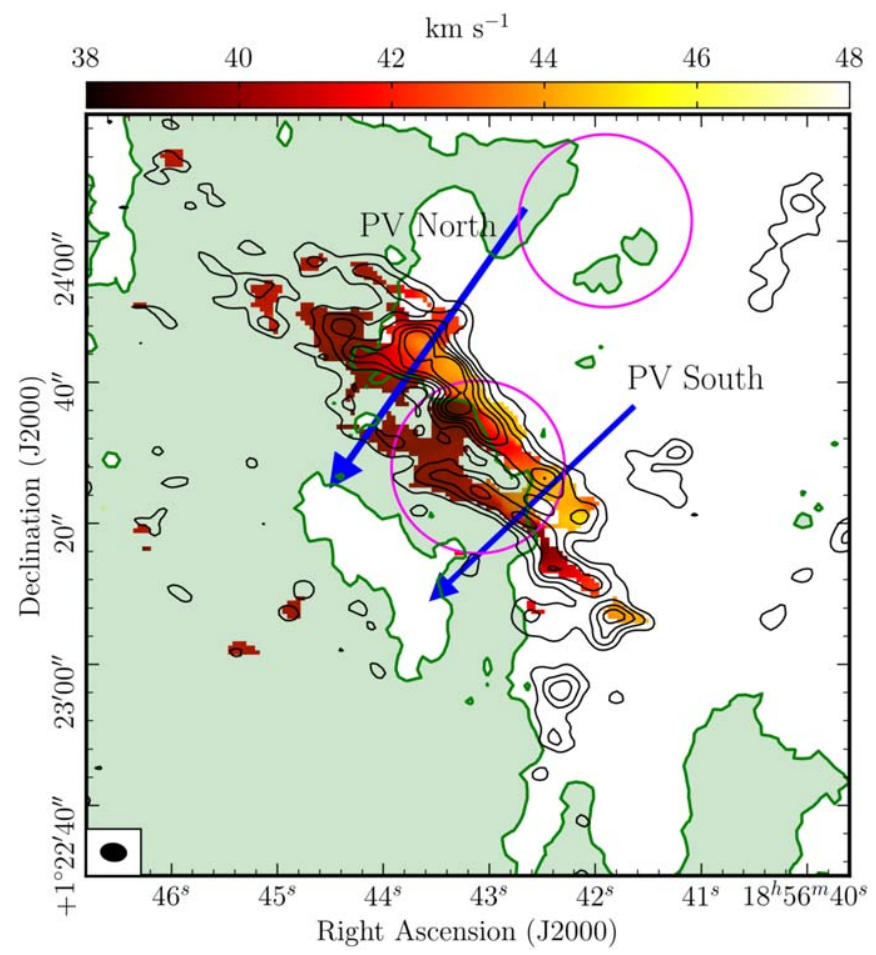

Figure 2. $\mathrm{SiO}$ integrated intensity map (black contours; velocity range 36.6 $-47.6 \mathrm{~km} \mathrm{~s}^{-1}$ ) toward G034 superimposed on its moment 1 velocity map (red scale). Contours are from $3 \sigma\left(\sigma=0.016 \mathrm{Jy} \mathrm{km} \mathrm{s}^{-1}\right)$ by steps of $3 \sigma$. Green contour and shadow indicates the $A_{v} \geqslant 20 \mathrm{mag}$ dense material in the IRDC (Kainulainen \& Tan 2013). Blue arrows show the directions used to extract the PV diagrams of Figure 4. Magenta circles indicate the two positions used to estimate the gas density conditions. Beam size is indicated as a black ellipse in the bottom left corner.

\section{2. $\mathrm{C}^{18} \mathrm{O}$ Single-dish Spectra: $\mathrm{H}_{2}$ Density and $\mathrm{SiO}$ Abundance Enhancement in the Post-shock Gas}

Figure 5 shows spectra of $\mathrm{C}^{18} \mathrm{O}(1-0)$ (black) and $\mathrm{C}^{18} \mathrm{O}(2-1)$ (blue) measured over a $25^{\prime \prime}$ beam toward two positions in G034, one outside and one inside the ridge (magenta circles in Figure 2). Outside the ridge, where no $\mathrm{SiO}$ is detected, only the quiescent (pre-shock) component at $\sim 43 \mathrm{~km} \mathrm{~s}^{-1}$ can be seen (Figure 2, bottom panel). However, inside the ridge, a second component at $\sim 40 \mathrm{~km} \mathrm{~s}^{-1}$ clearly appears coinciding with the detection of $\mathrm{SiO}$ (the post-shock gas; top panel).

By using the radiative transfer code RADEX (Van der Tak et al. 2007), we can infer the $\mathrm{H}_{2}$ gas volume density of the preshock $\left(\sim 43 \mathrm{~km} \mathrm{~s}^{-1}\right)$ and post-shock $\left(\sim 40 \mathrm{~km} \mathrm{~s}^{-1}\right)$ gas in G034 from the $\mathrm{C}^{18} \mathrm{O} J=1 \rightarrow 0$ and $J=2 \rightarrow 1$ emission. We assume a kinetic temperature of $15 \mathrm{~K}$ (typical of IRDCs; Pillai et al. 2007) and mean line width of $2 \mathrm{~km} \mathrm{~s}^{-1}$. The physical properties of the pre-shock gas at $43 \mathrm{~km} \mathrm{~s}^{-1}$ are $n\left(\mathrm{H}_{2}\right) \sim 10^{4} \mathrm{~cm}^{-3}, \quad N\left(\mathrm{C}^{18} \mathrm{O}\right) \sim 1.8 \times 10^{15} \mathrm{~cm}^{-2}, \quad \tau \sim 0.17$, and Tex $\sim 9 \mathrm{~K}$, while for the post-shock gas at $40 \mathrm{~km} \mathrm{~s}^{-1}$ the inferred values are $n\left(\mathrm{H}_{2}\right)>10^{5} \mathrm{~cm}^{-3}$, $N\left(\mathrm{C}^{18} \mathrm{O}\right) \sim 9 \times 10^{14} \mathrm{~cm}^{-2}, \tau \sim 0.36$, and $T_{\mathrm{ex}} \sim 7 \mathrm{~K}$. Hence, the density of the post-shock gas is a factor of $\geqslant 10$ higher than the density of the pre-shock gas. Note that the estimated excitation temperatures are comparable to those reported in Cosentino et al. (2018) for $\mathrm{CH}_{3} \mathrm{OH}$, another shock tracer.

We also estimate the $\mathrm{SiO}$ total column density, $N(\mathrm{SiO})$, for the pre-shock $\left(\sim 43 \mathrm{~km} \mathrm{~s}^{-1}\right)$ and post-shock $\left(\sim 40 \mathrm{~km} \mathrm{~s}^{-1}\right)$ gas. Since no $\mathrm{SiO}$ emission is present outside the ridge, the $3 \sigma$ upper limit for $N(\mathrm{SiO})$ in the pre-shock gas is $6 \times 10^{9} \mathrm{~cm}^{-2}$ by considering an rms noise level of $10 \mathrm{mJy}^{\mathrm{beam}}{ }^{-1}$. For the postshock gas, the derived $N(\mathrm{SiO})$ is $1.6 \times 10^{12} \mathrm{~cm}^{-2}$. Hence, the abundance of $\mathrm{SiO}$ has been enhanced by a factor $\geqslant 500$ in the post-shock gas in $\mathrm{G} 034$, as inferred from the $N(\mathrm{SiO}) / N\left(\mathrm{C}^{18} \mathrm{O}\right)$ column density ratios.

\section{3. $3 \mathrm{~mm}$ Continuum Emission}

From the $3 \sigma \mathrm{rms}$ level measured in our ALMA $3 \mathrm{~mm}$ continuum image $(0.2 \mathrm{mJy})$, we can estimate an upper limit to the envelope mass of any possible protostar present in the region as

$$
M_{\mathrm{env}}=\frac{F_{\nu} d^{2}}{B_{\nu}\left(T_{\mathrm{dust}}\right) k_{\nu}} \frac{M_{\mathrm{gas}}}{M_{\mathrm{dust}}},
$$

where $F_{\nu}$ is the continuum flux, $d$ is the source distance, $k_{\nu}$ is the dust opacity, $B_{\nu}\left(T_{\text {dust }}\right)$ is the Planck function at the dust temperature $T_{\text {dust }}$, and $M_{\mathrm{gas}} / M_{\text {dust }}$ is the gas-to-dust mass ratio (Ossenkopf \& Hennings 1994). We assume $d=2.9 \mathrm{kpc}$, $T_{\text {dust }}=15 \mathrm{~K}, \quad M_{\text {gas }} / M_{\text {dust }}=100$, and $F_{\nu}=0.2 \mathrm{mJy}$. Dust opacity at $3 \mathrm{~mm}$ is extrapolated to $k_{\nu}=0.0237 \mathrm{~m}^{2} \mathrm{~kg}^{-1}$ (Ossenkopf \& Hennings 1994). From all this, we derive a protostellar envelope mass $\leqslant 0.45 M_{\odot}$.

\section{Modeling the SiO PV diagrams}

We have used the 1D MHD shock code MHD_VODE (Flower \& Pineau de Forêts 2015) to reproduce the SiO velocity structure measured along the north and south PV diagrams in G034 and to constrain the physical parameters of the shock. We have run a grid of models with shock speeds between $v_{s}=5-30 \mathrm{~km} \mathrm{~s}^{-1}$, in accordance with the expanding and shocked velocities of the molecular gas in W44 (Sashida et al. 2013; Anderl et al. 2015b). We assume H pre-shock volume densities of $n(\mathrm{H})=10^{4}-10^{5} \mathrm{~cm}^{-3}$ (see Section 3.2) and consider cosmic-ray ionization rates $\zeta=1 \times 10^{-17}-5 \times 10^{-15} \mathrm{~s}^{-1}$ (Vaupre 2015), magnetic field strengths $B_{\text {mag }}=100-1000 \mu \mathrm{G}$ (Hoffman et al. 2005; Cardillo et al. 2014), and a pre-shock gas temperature of $15 \mathrm{~K}$ (Pillai et al. 2007; Ragan et al. 2011). The $v_{\mathrm{LSR}}$ of the pre-shock gas ranges from 45 to $50 \mathrm{~km} \mathrm{~s}^{-1}$, in agreement with the velocities measured outside W44 (Section 3.1 and Sashida et al. 2013).

As shown in Figure 4, the SiO PV diagrams are best reproduced by time-dependent CJ-type shocks that have not reached steady state yet and that are still evolving. The velocity profiles of the neutral and ion fluids $\left(v_{n}\right.$ and $\left.v_{i}\right)$ are shown by solid and dashed magenta lines, respectively, while the drift velocity, $\left|v_{i}-v_{n}\right|$, is shown by solid yellow lines. PV north can be fitted by a model with $n(\mathrm{H})=10^{4} \mathrm{~cm}^{-3}, v_{s}=22.5 \mathrm{~km} \mathrm{~s}^{-1}$, $\zeta=5 \times 10^{-16} \mathrm{~s}^{-1}, B_{\mathrm{mag}}=850 \mu \mathrm{G}$, and $t_{\mathrm{dyn}}=16,000 \mathrm{yr}$. The $\mathrm{SiO}$ emission appears at distances where the drift velocity $\left|v_{i}-v_{n}\right|>0$, consistent with the idea that $\mathrm{SiO}$ is released by dust erosion/destruction in the shock. However, the $\mathrm{SiO}$ column density measured in G034 $\left(1.6 \times 10^{12} \mathrm{~cm}^{-2}\right.$; Section 3.2) cannot be predicted by the models. This may be due to the fact that the public version of the MHD_VODE code does not include grain-grain collision processes such as shattering. While the threshold drift velocities for the production of $\mathrm{SiO}$ by sputtering are $>20 \mathrm{~km} \mathrm{~s}^{-1}$ (Gusdorf et al. 2008; Jimenez-Serra et al. 2008), shattering only requires velocities as low as $1 \mathrm{~km} \mathrm{~s}^{-1}$ (Guillet et al. 2011). The MHD_VODE code does not consider a small fraction of $\mathrm{SiO}$ in the dust mantles either, which may also alleviate the 


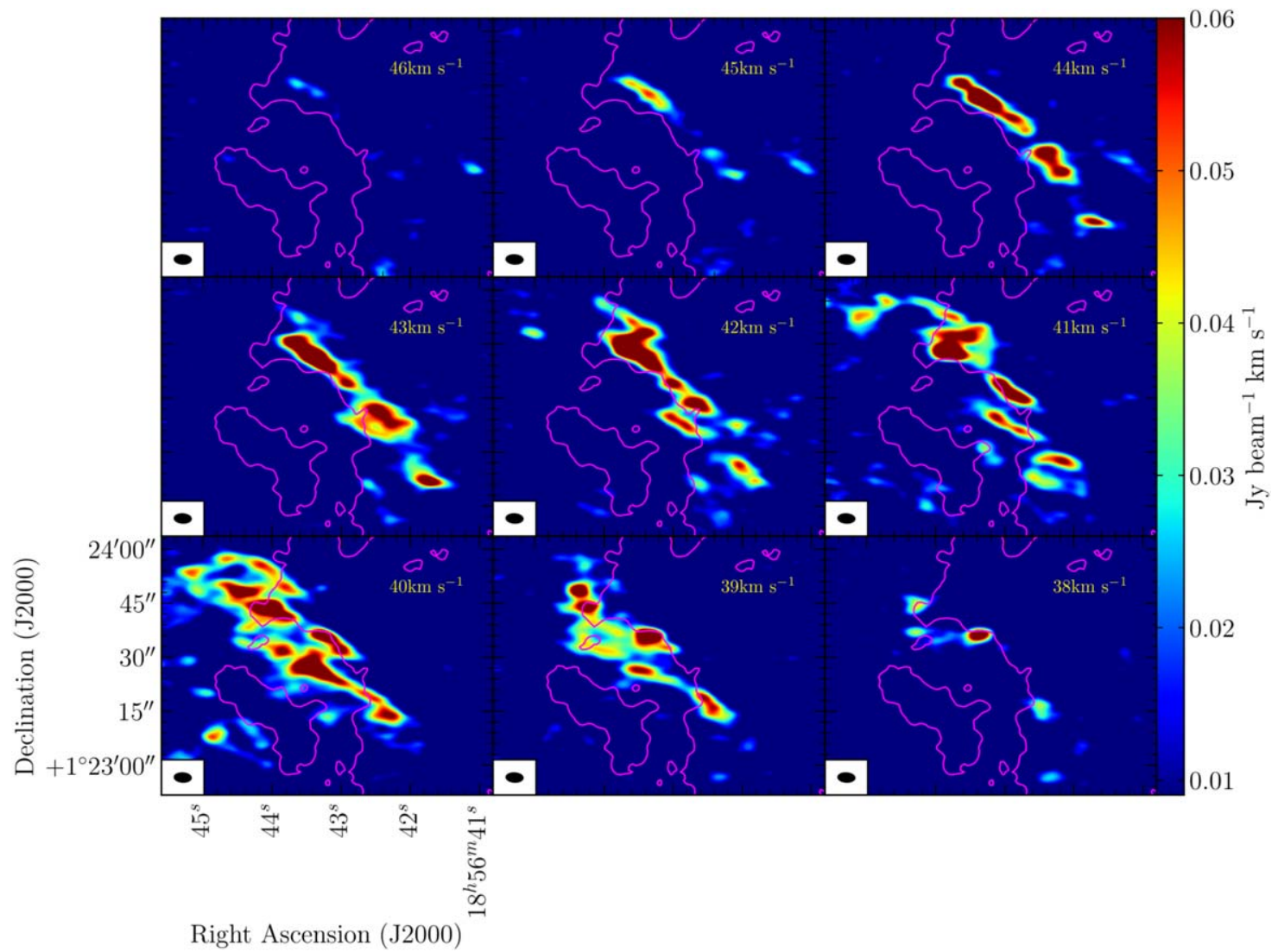

Figure 3. $\mathrm{SiO}$ channel maps for the velocity range $38-46 \mathrm{~km} \mathrm{~s}^{-1}$. Integration step is $1 \mathrm{~km} \mathrm{~s}^{-1}$, and intensities are $\geqslant 3 \sigma\left(\sigma=0.006 \mathrm{Jy} \mathrm{beam}^{-1} \mathrm{~km} \mathrm{~s}^{-1}\right)$. The magenta contour (with $A_{\mathrm{v}} \sim 20 \mathrm{mag}$; Kainulainen \& Tan 2013) indicates the position of the high-density ridge found toward the northwest of G034. Beam size and central velocity are shown for each map.

discrepancy between the observed and predicted $\mathrm{SiO}$ column densities (see, e.g., Schilke et al. 1997; Jimenez-Serra et al. 2008)

As expected from the interaction with an SNR (Hoffman et al. 2005; Cardillo et al. 2014), the inferred value of $B_{\mathrm{mag}}$ in PV north is higher than those measured in molecular clouds with $n\left(\mathrm{H}_{2}\right) \sim 10^{4}-10^{5} \mathrm{~cm}^{-3}$ (Critcher et al. 2010). In fact, only models with such large $B_{\text {mag }}$ values can predict long dissipation lengths as observed in PV north.

In contrast to PV north, no model can reproduce well the sharp decrease in velocity observed in PV south. The best-fit model gives $\quad n(\mathrm{H})=10^{4} \mathrm{~cm}^{-3}, \quad v_{s}=23 \mathrm{~km} \mathrm{~s}^{-1}$, $\zeta=1 \times 10^{-15} \mathrm{~s}^{-1}, \quad B_{\mathrm{mag}}=880 \mu \mathrm{G}$, and $17,000 \mathrm{yr}$. This mismatch between the model and the observations could be due to $\mathrm{SiO}$ (a high-density tracer) not being excited enough along the whole length of the shock. Alternatively, MHD shock theory may need to be revisited.

\section{Discussion}

\subsection{SiO Emission Caused by Protostars and Outflows?}

Our ALMA $3 \mathrm{~mm}$ continuum map does not reveal the presence of any pointlike source in G034 associated with the $\mathrm{SiO}$ emission down to a protostellar envelope mass $0.45 M_{\odot}$ (Section 3.3). This value is comparable to the lower limits of Class 0 and I envelope masses ( $>0.5 M_{\odot}$; Arce \& Sargent 2006) and well below the typical values for high-mass protostars (>100 $M_{\odot}$; Zhang et al. 2005). Therefore, if present, both lowmass and high-mass protostars should have been detected in our $3 \mathrm{~mm}$ ALMA image.
In addition, following Dierickx et al. (2015), we have estimated the mass $(M)$, linear momentum $(P)$, and kinetic energy $(E)$ of the $\mathrm{SiO}$ emission in $\mathrm{G} 034$ to be $M=12.2 M_{\odot}$, $P=18.8 M_{\odot} \mathrm{km} \mathrm{s}^{-1}$, and $E=3.8 \times 10^{41} \mathrm{erg}$. Outflows driven by low-mass protostars have typical parameters in the ranges $M \sim 0.005-0.15 M_{\odot}, P \sim 0.004-0.12 M_{\odot} \mathrm{km} \mathrm{s}^{-1}$, and $E \sim 2 \times 10^{40}-5 \times 10^{42} \mathrm{erg}$ (Arce \& Sargent 2006). Although the kinetic energy inferred from $\mathrm{SiO}$ is comparable to that in outflows driven by low-mass protostars, the mass and linear momentum are factors 100-3000 higher. Outflows driven by high-mass protostars have masses of a few tens of $M_{\odot}$ and linear momentum $\sim 10-100 M_{\odot} \mathrm{km} \mathrm{s}^{-1}$, comparable to our values (Zhang et al. 2005), but their kinetic energies are 5 orders of magnitude higher than measured in G034 $\left(10^{46} \mathrm{erg}\right.$ versus $3.8 \times 10^{41} \mathrm{erg}$; Zhang et al. 2005). It is thus unlikely that $\mathrm{SiO}$ in $\mathrm{G034}$ is associated with star formation activity.

\subsection{A Molecular Flow-Flow Collision Unveiled by Plunging Waves}

G034 presents little star formation activity (Rathborne et al. 2006; Chambers et al. 2009), and hence internal stellar feedback is not expected to affect the pristine physical and chemical conditions of the cloud. In such a quiescent region, molecular shock tracers such as $\mathrm{SiO}$ are heavily depleted onto dust grains (Martín-Pintado et al. 1992; Schilke et al. 1997). Cosentino et al. (2018), however, reported the presence of widespread and narrow $\mathrm{SiO}$ emission (average line width of $1.6 \mathrm{~km} \mathrm{~s}^{-1}$ ) toward the northwest of $\mathrm{G} 034$ at an angular resolution $\sim 30^{\prime \prime}$. 

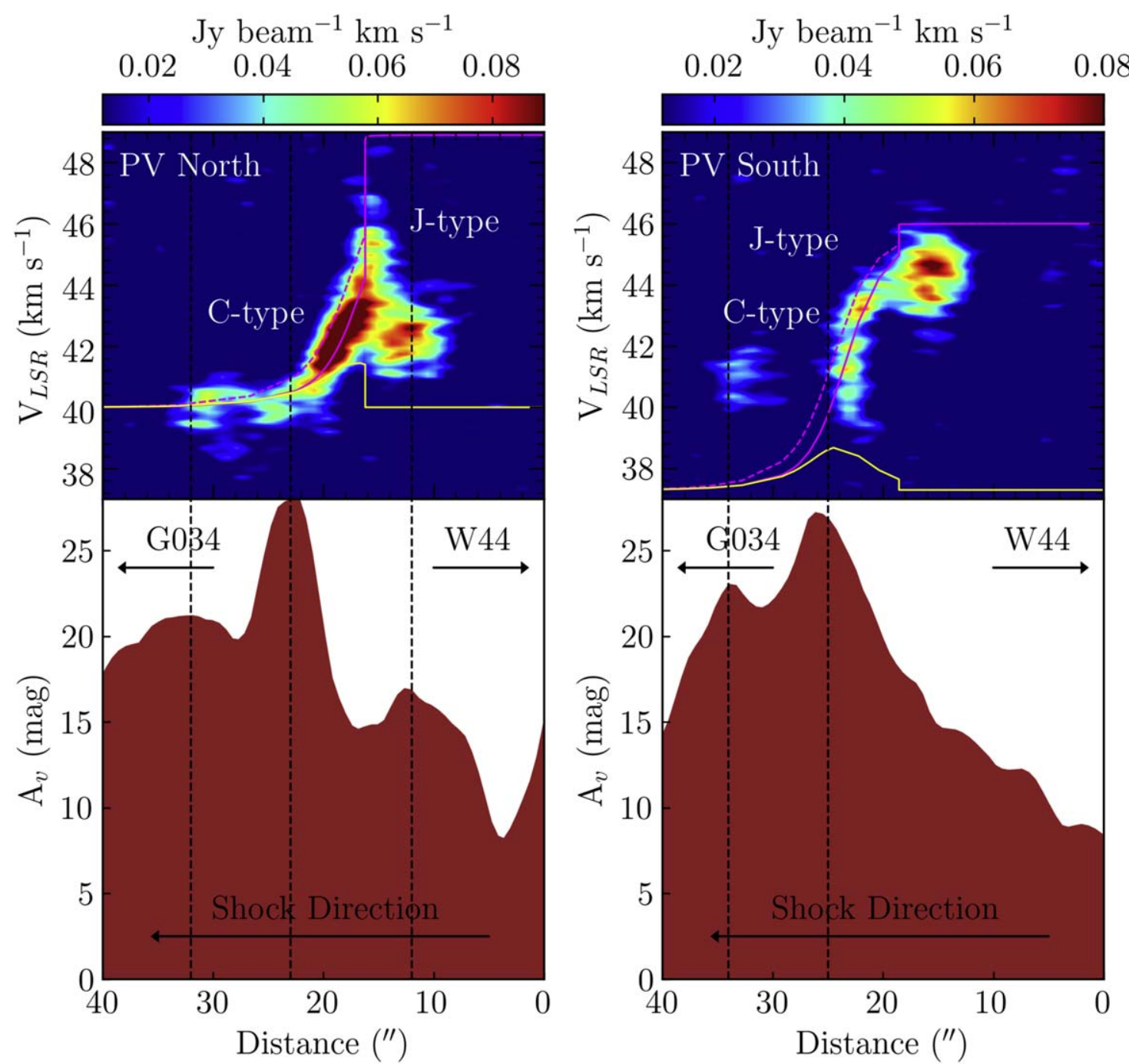

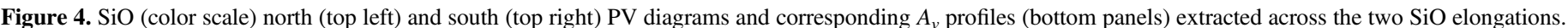

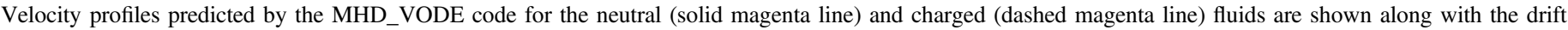
velocity $\left(\left|v_{i}-v_{n}\right|\right)$ between the two fluids (yellow line). Vertical dashed lines (in black) show the local peaks in extinction.

Our ALMA images reveal that $\mathrm{SiO}$ in $\mathrm{G} 034$ is organized into two plane-parallel structures that seem to impact onto the northwestern dense ridge of G034. In a way analog to a sea wave plunging onto a shore, the molecular gas pushed away by the W44 SNR (the sea wave) encounters the denser material of the IRDC (the beach), plunges, and breaks causing the formation of two plane-parallel wavefronts or MHD shocks seen in $\mathrm{SiO}$. As shown in Section 4, such a kinematic structure is best reproduced by a nonstationary CJ-type MHD shock, where the J-type discontinuity is seen in the PV diagrams as an almost vertical velocity drop, and the C-type part shows a shallower deceleration over $\sim 10^{\prime \prime}$. The different velocity shapes of the $\mathrm{SiO}$ north and south $\mathrm{PV}$ diagrams may reflect inhomogeneities in the gas and/or slightly different physical conditions of the pre-shock gas (the derived $\zeta$ differ by a factor of 2 between PV north and south). The proximity of W44 is responsible for the high $\zeta$ and $B_{\text {mag }}$ required by the models.

The dynamical ages predicted for the shocks in PV north and PV south $\left(t_{\mathrm{dyn}}=16,000-17,000 \mathrm{yr}\right)$ are in agreement with the age of the SNR ( 20,000 yr) and the $\mathrm{SiO}$ depletion timescales $\left(\sim 10^{4}-10^{5}\right.$ yr; Martín-Pintado et al. 1992). We also caution that the MHD_VODE code only provides 1D highly idealized models that do not consider projection effects, which may explain any disagreement between the model predictions and the observations (especially for PV south).

Finally, we propose that the shock interaction in G034 between the expanding molecular gas from W44 and the preexisting IRDC may have shaped the $A_{\mathrm{v}} \geqslant 20$ mag ridge into its filamentary morphology, since the latter appears as an almost detached structure from the IRDC and since the $\mathrm{SiO}$ emission follows the shape of the ridge.

This is supported by the fact that the density of the postshock component in G034 is a factor $>10$ higher than in the pre-shock gas $\left(n\left(\mathrm{H}_{2}\right)>10^{5} \mathrm{~cm}^{-3}\right.$; Section 3.2), which may enable star formation. The lack of any cold core in the ridge is consistent with the idea that we are observing the earliest stages of such a process, since the age of the shock $(<20,000 \mathrm{yr})$ is too short to see the effects of gravitational collapse of any newly formed high-density structure or core (timescales $\sim 10^{5} \mathrm{yr}$ for densities of $n\left(\mathrm{H}_{2}\right)>10^{5} \mathrm{~cm}^{-3}$; McKee \& Tan 2003).

In summary, we report the first detection of a fully resolved, time-dependent CJ-type MHD shock. This shock has been produced by the interaction between a molecular flow expanding from the SNR W44 and a high-density ridge part 

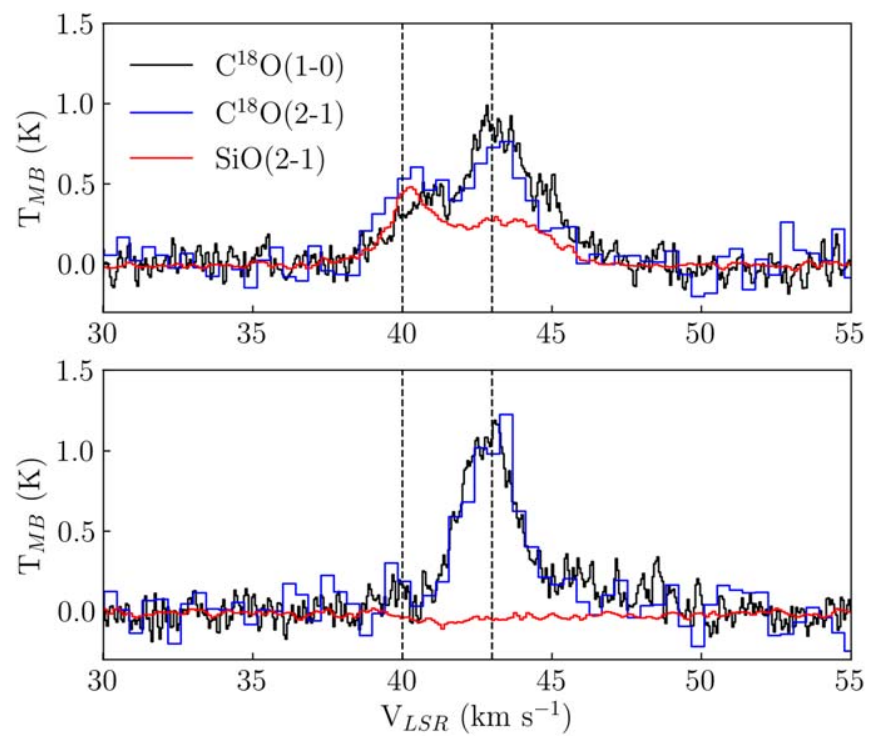

Figure 5. Spectra of $\mathrm{C}^{18} \mathrm{O}(1-0)$ (black lines), $\mathrm{C}^{18} \mathrm{O}(2-1)$ (blue lines), and $\mathrm{SiO}$ $(2-1)$ (red lines) extracted toward G034 at positions $\alpha(\mathrm{J} 2000)=18^{\mathrm{h}} 56^{\mathrm{m}} 43^{\mathrm{s}} .1$, $\delta(\mathrm{J} 2000)=1^{\circ} 23^{\prime} 28^{\prime \prime}$ (top panel) and $\alpha(\mathrm{J} 2000)=18^{\mathrm{h}} 56^{\mathrm{m}} 41.9^{\mathrm{s}}, \delta(\mathrm{J} 2000)=1^{\circ}$ $24^{\prime} 03^{\prime \prime}$ over a $25^{\prime \prime}$ beam. The components at $43^{\mathrm{s}}$ and $40 \mathrm{~km} \mathrm{~s}^{-1}$ corresponds to pre-shock and post-shock gas, respectively (vertical dashed lines).

of the nearby IRDC G034.77-00.55. The high magnetic field and cosmic-ray ionization rate induced by the SNR is responsible for the large dissipation region of the CJ-type shock. The post-shock gas is compressed by the shock to densities $n(\mathrm{H})>10^{5} \mathrm{~cm}^{-3}$, comparable to those required for the formation of massive stars.

This Letter makes use of the ALMA data: ADS/JAO. ALMA \#2016.1.01363.S. ALMA is a partnership of ESO (representing its member states), NSF (USA) and NINS (Japan), together with NRC (Canada), MOST and ASIAA (Taiwan), and KASI (Republic of Korea), in cooperation with the Republic of Chile. The Joint ALMA Observatory is operated by ESO, AUI/NRAO and NAOJ. This Letter also makes use of observations carried out with the IRAM $30 \mathrm{~m}$ telescope under projects $025-12$. IRAM is supported by INSU/ CNRS (France), MPG (Germany), and IGN (Spain). G.C. acknowledges financial support from University College London Perren/Impact Studentship and from the ESO Studentship Program Europe 2017/2018. I.J.-S. acknowledges the support from the STFC through an Ernest Rutherford Fellowship (grant No. ST/L004801), and from the MINECO and FEDER funding under grant ESP2017-86582-C4-1-R. A.T.B acknowledges funding from the European Union Horizon 2020 research and innovation programme (grant agreement No. 726384).

\section{ORCID iDs}

Giuliana Cosentino (iD https://orcid.org/0000-0001-5551-9502 Izaskun Jiménez-Serra (i) https://orcid.org/0000-00034493-8714

Paola Caselli (iD https://orcid.org/0000-0003-1481-7911

Jonathan D. Henshaw (1D https://orcid.org/0000-0001-

9656-7682
Jonathan C. Tan (1D https://orcid.org/0000-0002-3389-9142

Serena Viti (D) https://orcid.org/0000-0001-8504-8844

Francesco Fontani (i) https://orcid.org/0000-0003-0348-3418

Benjamin Wu (iD https://orcid.org/0000-0003-3874-7030

\section{References}

Anderl, S., Gusdorf, A., \& Güsten, R. 2015a, A\&A, 569, A81 Anderl, S., Gusdorf, A., \& Güsten, R. 2015b, PoS, arXiv:1503.06586 Arce, H. G., \& Sargent, A. I. 2006, ApJ, 646, 1070 Babel, J., \& Montmerle, T. 1997, A\&A, 323, 121

Beuther, H., Bihr, S., Rugel, M., et al. 2016, A\&A, 595, A32

Cardillo, M., Tavani, M., Giuliani, A., et al. 2014, A\&A, 565, A74

Carey, S. J., Noriega-Crespo, A., Mizuno, D. R., et al. 2009, PASP, 121, 76

Caselli, P., Hartquist, T. W., \& Havnes, O. 1997, A\&A, 322, 296

Chambers, E. T., Jackson, J. M., Rathborne, J. M., \& Simon, R. 2009, ApJS, 181,360

Chiéze, J.-P., Pineau de Forêts, G., \& Flower, D. R. 1998, MNRAS, 295, 672

Churchwell, E., Babler, B. L., Meade, M. R., et al. 2009, PASP, 121, 213

Claussen, M. J., Frail, D. A., Goss, W. M., et al. 1997, ApJ, 489, 143

Cosentino, G., Jiménez-Serra, I., Henshaw, J. D., et al. 2018, MNRAS, 474, 3760

Critcher, R., Wandelt, B., Heiles, C., Falgarone, E., \& Troland, T. H. 2010, ApJ, 725, 466

Dierickx, M., Jiménez-Serra, I., Rivilla, V. M., \& Zhang, Q. 2015, ApJ, 803, 89

Draine, B. T. 1980, ApJ, 241, 1021

Draine, B. T., Roberge, W. A., \& Dalgarno, A. 1983, ApJ, 264, 485

Flower, D. R., \& Pineau de Forêts, G. 2003, MNRAS, 343, 390

Flower, D. R., \& Pineau de Forêts, G. 2015, A\&A, 578, A63

Girart, J. M., \& Acord, J. M. P. 2001, ApJL, 552, L63

Gotthelf, E. V., Koralesky, B., Rudnick, L., et al. 2001, ApJL, 552, L39

Grefenstette, B. W., Harrison, F. A., Boggs, S. E., et al. 2014, Natur, 506, 339

Guillet, V., Pineau de Forêts, G., \& Jones, A. P. 2011, A\&A, 527, A123

Gusdorf, A., Pineau de Forêts, G., Cabrit, S., \& Flower, D. R. 2008, A\&A, 490, 695

Hoffman, I. M., Goss, W. M., Brogan, C. L., \& Claussen, M. J. 2005, ApJ, $627,803 \mathrm{H}$

Inutsuka, S., Inoue, T., Iwasaki, K., \& Hosokawa, T. 2015, A\&A, 580A, A49

Jimenez-Serra, I., Caselli, P., Martín-Pintado, J., \& Hartquist, T. W. 2008, A\&A, 482, 549

Kainulainen, J., \& Tan, J. C. 2013, A\&A, 549, A53

Lesaffre, P., Chiéze, J.-P., Cabrit, S., \& Pineau de Forêts, G. 2004, A\&A, 427, 157

Martín-Pintado, J., Bachiller, R., \& Fuente, A. 1992, A\&A, 254, 315

McKee, C. F., \& Tan, J. C. 2003, ApJ, 585, 850

Miceli, M., Orlando, S., Burrows, D. N., et al. 2019, NatAs, 2, 236

Neuufeld, D. A., \& Dalgarno, A. 1989, ApJ, 340, 869

Ortega, M. E., Cichowolski, S., Castelletti, G., \& Dubner, G. 2007, BAAA, 50,189

Ossenkopf, V., \& Hennings, T. 1994, A\&A, 291, 943

Pillai, T., Wyrowski, F., Hatchell, J., Gibb, A. G., \& Thompson, M. A. 2007, A\&A, 467, 207

Ragan, S. E., Bergin, E. A., \& Wilner, D. 2011, ApJ, 736, 163

Ranasinghe, S., \& Leahy, D. A. 2018, AJ, 155, 204

Rathborne, J. M., Jackson, J. M., \& Simon, R. 2006, ApJ, 641, 389

Sashida, T., Oka, T., Tanaka, K., et al. 2013, ApJ, 774, 7

Schilke, P., Walmsley, C. M., Pineau de Forêts, G., \& Flower, D. R. 1997, A\&A, 321, 293

Seta, M., Hasegawa, T., Sakamoto, S., et al. 2004, AJ, 127, 1098

Seta, M., Winnewisser, G., Hasegawa, T., \& Oka, T. 1998, ApJ, 505, 286

Van der Tak, F. F. S., Black, J. H., Schöier, F. L., Jansen, D. J., \& van Dishoeck, E. F. 2007, A\&A, 468, 627

Vaupre, S. 2015, PhD thesis, Univ. Grenoble Alpes

Wilkin, F. P. 1996, ApJL, 459, L31

Wu, B., van Loo, S., Tan, J. C., \& Bruderer, S. 2015, ApJ, 811, 56

Yoshiike, S., Fukuda, T., Sano, H., et al. 2013, ApJ, 768, 179

Zhang, Q., Hunter, T. R., Brand, J., et al. 2005, ApJ, 625, 864 